\title{
Optical Multistability and Oscillations in Hybrid Optical Bistable Systems with Short Delay Times
}

\author{
M.A. Muriel and J.A. Martín-Pereda \\ E.T.S. Ingenieros de Telecomunicación, U.P.M., Ciudad Universitaria, \\ E-28040 Madrid, Spain
}

\section{INTRODUCTION}

Optical instabilities in the output light from a bistable optical device (BOD) with a delayed feedback was predicted by Ikeda [1]. Gibbs et a]. [2] gave the first experimental verification of this tyne of instabilities. From that time several groups have studied the instabilities of the BOD for different relations between the delay time $t_{R}$ and the time constant $\tau$ of the system. In a previous paver [3] an empirical and analytical study of instabilities in hybrid BOD was reported by us. The employed set up is shown in Fig. 1.

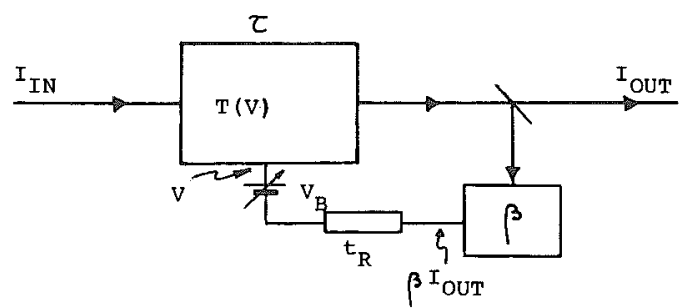

Fia. 1

In this paper, and in order to verify the theory, a trianaular voltage bias was added to the feedback instead of the usual constant one. In this way the working line is moving with the same slope, $\mathrm{tg}^{-1}$ BI IN , back and forth periodically, $\beta$ being the feedback factor and I $I_{N}$ the input 7 ight. The transmission slope $\frac{d T}{d V}$ was taken, for a constant input, negative. The obtained output was periodic oscillations if the delay time $t_{R}$ was larger than the material response time The general expressions giving the possible oscillating frequencies were

$$
\begin{aligned}
& \left|\beta I_{I N}\left(\frac{d T}{d V}\right)_{\bar{V}}\right|=\sqrt{1+(\omega \tau)^{2}} \\
& \operatorname{tg}\left(\omega t_{R}\right)=-\omega \tau
\end{aligned}
$$

being $\bar{V}$, the voltage correspondino to the equilibrium point and $V_{B}$ the bias voltage.

Because the time $₹$ was very short compared with $t_{R}$, was taken as zero for the rest of the paper [3].

In this paper an extension of the previous reported theory will be given. Our study will cover the results for a feedback delay time $t_{B}$ shorter that the material response time $\tau$ being the transmission slope either negative or positive. 
From equations (1) and (2), if time $\tau$ is no longer zero, will be possible to obtain the situation at the equilibrium point. In general the onset of instabilities is given by

$$
\frac{d y_{n}}{d y_{n-1}} \leqslant-1
$$

where $\frac{d y_{n}}{d y_{n-1}}$ is the single step amplification rate. In our case this expression is equivalent to

$$
\left(\frac{d T}{d V}\right)_{\vec{V}} \leqslant-\frac{1}{\beta I_{I N}}
$$

This expression chanaes now to

$$
-\infty \leqslant\left(\frac{d T}{d V}\right)_{\bar{V}} \leqslant-\frac{\sqrt{1+(\omega \tau)^{2}}}{\beta I_{I N}}
$$

This is the oscillation condition in the $T(V)$ vs. $V$ plane and can be extended for $I_{\text {OUT }}$ vs. I IN and $\left(\beta I_{0 U T}\right)$ vs. $V_{B}$ planes. In the first case the result is

I OUT

$$
0 \leqslant \frac{d I_{\text {OUT }}}{d I_{I N}} \leqslant \frac{T}{1+\sqrt{1+(\omega \tau)^{2}}}
$$

For we very large, equations (5), (6) and (7) give just one value for oscillatory behaviour. These values are given by

$$
\left(\frac{d T}{d V}\right)_{V}=-\infty \quad ; \quad \frac{d I_{\text {OUT }}}{d I_{I N}}=0 ; \frac{d\left(\beta I_{\text {OUT }}\right)}{d V_{B}}=-1
$$

corresponding to a transmission curve perpendicular to the $V$ axis. This theoretical situation is very difficult to obtain in a real case because abrupt changes in the transmission characteristics are not common in most materials.

In order to obtain the oscillating frequency values, equation (2) has to be employed. This transcendenta $7^{\circ}$ equation can be solved graphically giving the following frequencies $\nu=\frac{1}{4 t_{R}}, \frac{5}{4 t_{R}}, \frac{9}{4 t_{R}}, \ldots \ldots$, because $t_{R}$ is very small these frequencies get very large values. These results are equivalents to previous studies in this field [4], [5].

\section{MULTISTABILITY BEHAVIOUR}

If a transmission curve with positive slope is taken now, hysteresis cycles are obtained instead the previous oscillations. As it will be shown and applying 


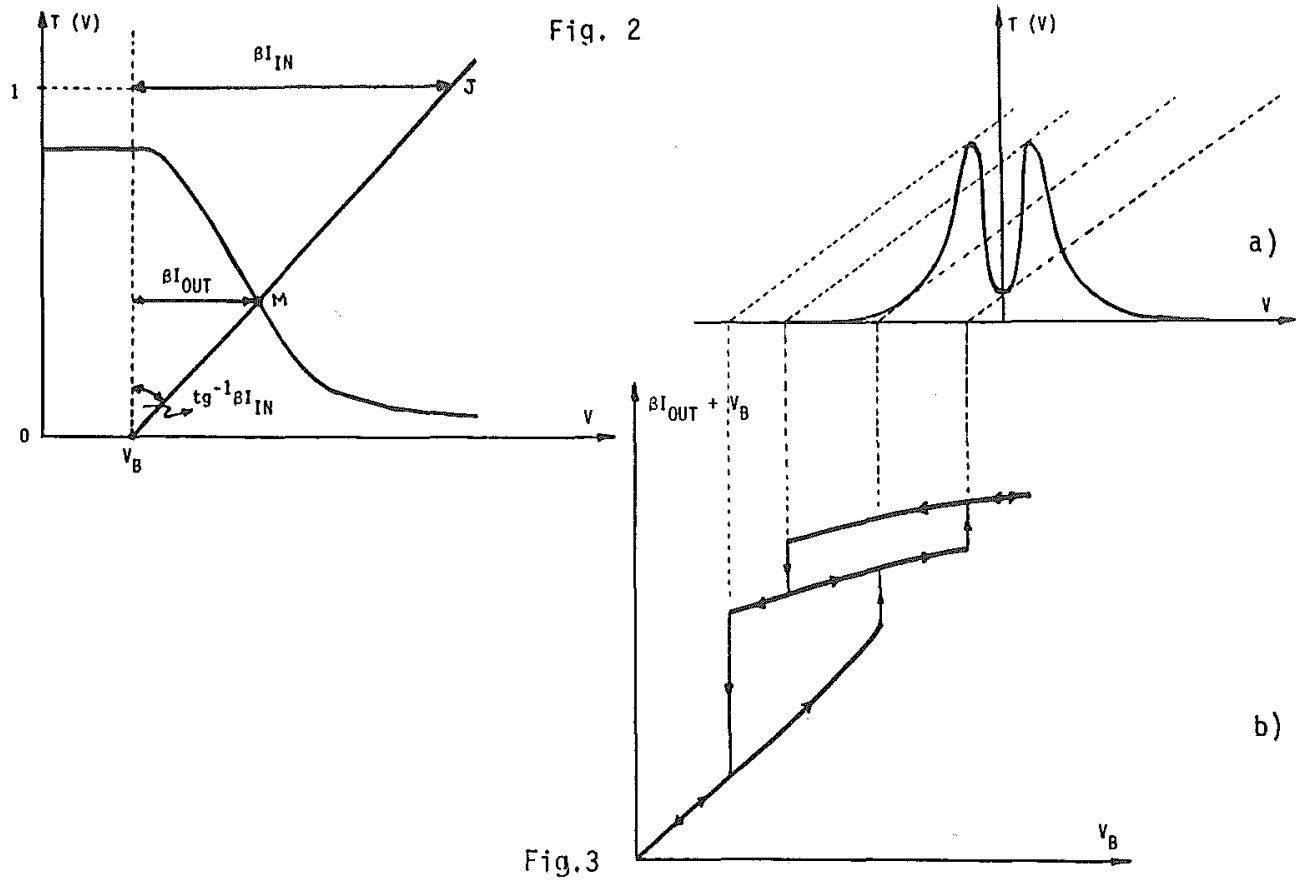

the triangular voltage indicated in paper [3] several types of cycles will be obtained.

In order to explain the results it is necessary to extend our previous geometrical interpretation of the working point in BOD's as given in [3]. In this way, as it can be obtained from Figure 2, the distance from the working point, $M$, to the $T$ axis is $\beta I_{0 U T}$ and from $J$ to the same axis $\beta I_{I N}$. These values can be easily obtained

just from Thales theorem.

In order to obtain empirically different conditions for the above facts, a similar set-up to the previously reported was employed [3]. In this way a twisted nematic liquid crystal cell has been used as nonlinear material. Depending on polarizers, relative orientations and cell positions, different transmission curves can be obtained.

The analytical results for the transmission curve shown in Fig. 3a are aiven in Fig. 3b. Two experimental results are shown in Figs. 4 and 5 for different configurations. As it can be verified these results are in perfect agreement with the geometrical results. Optical multistability is shown. This result offers the possibility to employ this tybe of system in optical non-binary logics.

\section{CONCLUSION}

A unified model for the optical behaviour in hybrid BOD's has been reported. This model can be summarized in Fig. 6, where the results for an ideal transmission curve are presented depending on the transmission slope. Region A was studied in the second part of this paper and zone $A^{\prime}$ in the first part and in paper [3].

Financial support from Spanish CAICYT (Grant 1564-82) is appreciated. 


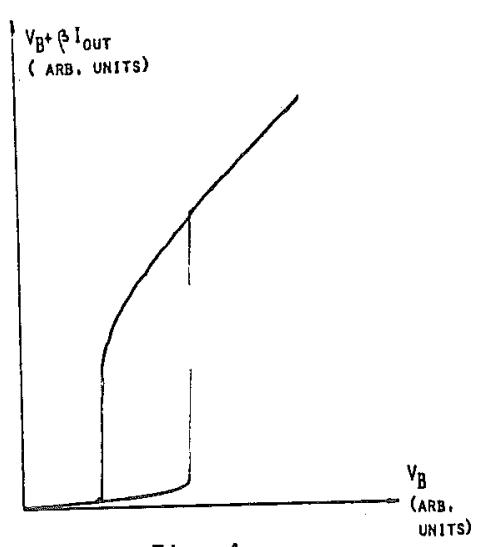

Fig. 4

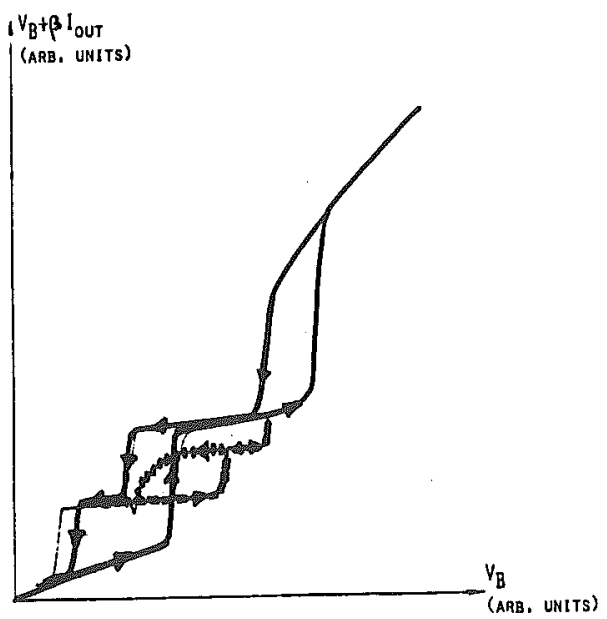

Fị. 5

(ARB, UNITS)
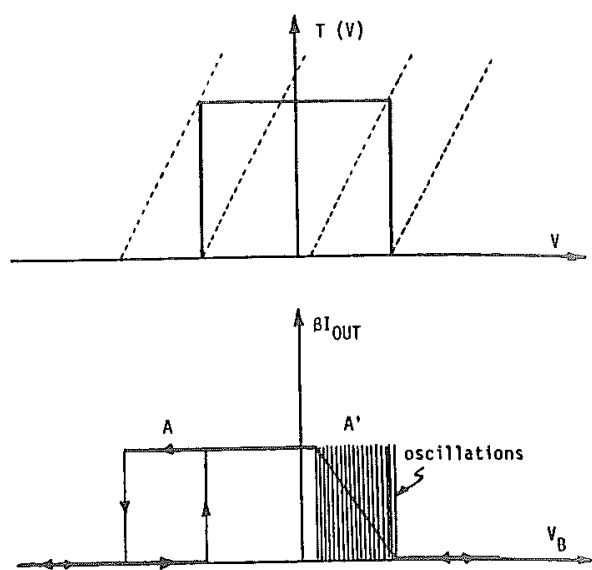

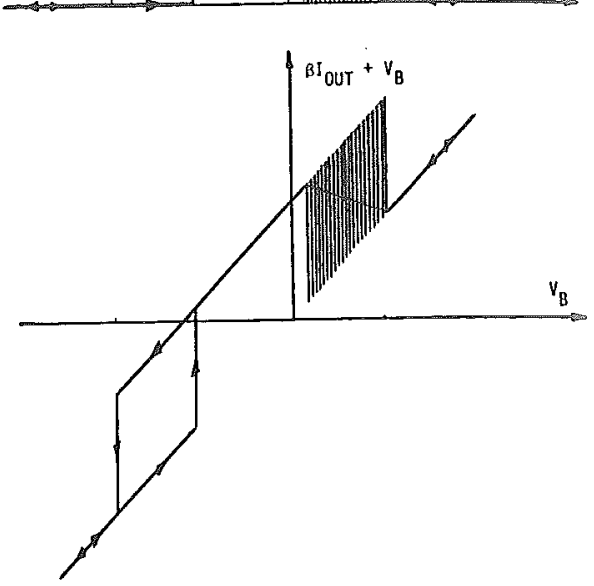

Fig. 6

\section{REFERENCES}

1. K. Ikeda, H.Daido and 0. Akimoto: Phys.Rev.Lett. 45, 709 (1980).

2. H.M. Gibbs, F.A. Hopf, D.L. Kaplan and R.L. Shoemaker: Phys.Rev.Lett.46, 474(1981).

3. J.A. Martín-Pereda and M.A. Muriel: Optical Bistability 2 p. 143 (P1eñum Press, New York, 1984).

4. P.M. Petersen, J.N. Ravn and T. Skettrup: IEEE J. Quant. Electr. 20, 690 (1984).

5. J.Y. Gao, J.M. Yuan and L.M. Narducci: Opt. Comm. 44, 201 (1983). 